\title{
The Study of EHD Flow:Velocity and electrical power
}

\author{
Sumariyah $^{1^{*}}$, Kusminarto ${ }^{2}$, Arief Hermanto ${ }^{2,}$ and Pekik Nuswantoro ${ }^{2}$ \\ ${ }^{I}$ Physics Department, Faculty of Sciences and Mathematics, Diponegoro University, Indonesia \\ ${ }^{2}$ Physics Department, Faculty of Mathematics and Natural Sciences, Gadjah Mada University, Indonesia
}

\begin{abstract}
Electrohydrodynamics (EHD) flow is the yield of the corona discharge. Corona discharge characterized by electrical phenomena that occur together in a gas medium under the influence of an electric field. In corona discharge, the electric field is not homogeneous due to the geometry of the electrode configuration. In this study, a pin-multi concentric ring electrode (P-MRE) and pin-single ring electrode (P-SRE) has been used as electrodes configuration. The objective of this research is to get the velocity of EHD flow and determining the electrical power of the EHD flow. EHD flow generated by a DC high voltage (up to $5 \mathrm{kV}$ ). The pin electrode was connected to a positive polarity and electrode ring/multi-concentric rings to negative polarity. Based on our result, we make a comparison of two types of corona configurations. The results were obtained that, EHD flow velocities with the PMRE was greater than the P-SRE for the same distance and voltage. We found that $\mathrm{u}-\mathrm{P}$ characteristics of EHD flow with the P-MRE and P-SRE follow relationship as $\mathrm{u} \infty \mathrm{P}^{1 / 3}$.
\end{abstract}

\section{Introduction}

Electrohydrodynamics (EHD) flow is the yield of corona discharge which is characterized by electrical phenomena. It occurs together in a gas medium under the influence of an electric field that is not homogeneous due to the geometry of the electrode configuration. The electric field will be very high in the area around the active electrode thereby allowing to happen ionization and the formation of high-energy electrons in these area. Around the active electrode the primer ionization process occurs and formed plasma [1]. The plasma surrounds part of the active electrode is usually shaped tapered so that the plasma resembles a corona [2]

The process of the incandescent corona discharge in an electrical field begins with Townsend discharge followed by glow discharge or corona discharge and ends with arc discharge. A Townsend discharge occurs at lower voltages where small number of ionizations occur. While at the time of arc discharge, the raised electric current very quickly followed by the very drop voltage sharply [3].

The prospects of EHD flow in the industry are quite large, such as for drug delivery systems that was developed by Repanas [4] and Sofokleous [5]. The study of EHD flow used as the coolant was carried out by Chen [6] and Hosseinloo [7]. The application of EHD as a drying apparatus was conducted by Bardy [8], Martynenko [9] and Karam [10]. Devices that use corona discharge EHD flow has many advantages such as: free of mechanical vibration and acoustic noise. In addition, the required electric power of EHD corona discharge is quite low [11].

\section{Research Procedure}

\footnotetext{
Corresponding author: sumariyah@undip.ac.id
}

Two configurations of the EHD flow generator were studied, they were a pin-multi concentric ring (P-MRE) and pin-single ring electrode (P-SRE) which was as the control of measurement. Pin electrodes made of stainless steel sewing needle with a diameter of pointed end 0.09 $\mathrm{mm}$. Multi concentric ring electrodes consist of 3 concentric ring electrodes are arranged concentrically interconnected. The third ring of the electrode constituent having diameters in each of $8 \mathrm{~mm}, 16 \mathrm{~mm}$ and $24 \mathrm{~mm}$, respectively. In a single ring electrode, the diameter was $24 \mathrm{~mm}$. All electrode metal rings have the same width and thickness, which were $2 \mathrm{~mm}$. The composition of the generating system of EHD flow in this study includes P-MRE, P-SRE, (high voltage power, set voltage and current measurement tools and a set of measuring tools EHD flow velocity). The air can come from the top and out of the bottom, as shown in Figure.1

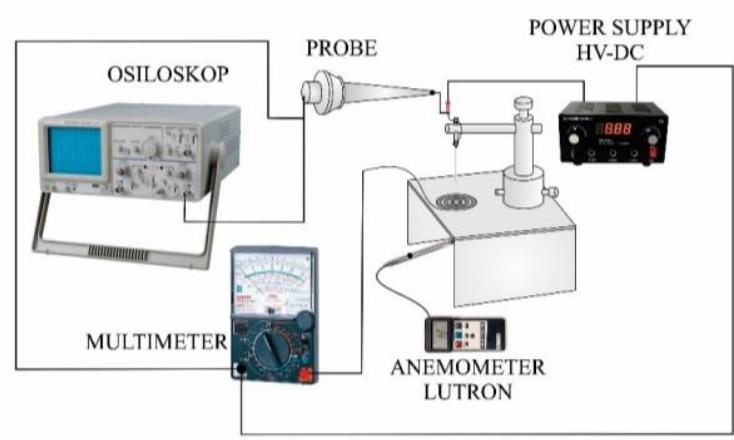

Fig.1.The Composition of a Generating System of the EHD Flow

The EHD flow generated by installing a high voltage DC $10 \mathrm{KV}$ on the electrodes of the corona discharge system with a positive polarity on the pin electrodes and a negative polarity on the ring/ the multiconcentric ring. A high-voltage probe 1000: 1 was used 
to measure the high voltage, which is connected to the CRO. The ion current was measured by using a multimeter. To Measure the velocity of EHD flow, a Hot-Wire Anemometer was placed perpendicular to the pin electrode direction with the distance was $15 \mathrm{~mm}$.

This method is the same as the method of our previous research. They were a study of EHD flow about modeling of electric potential distribution in the zone of EHD flow [12], measurement of the velocity of EHD flow [13] and determination of ion mobility in the zone of EHD flow [14].

\section{Results and Discussion}

\section{1 u-V Characteristic of EHD Flow}

Graph of the velocity of EHD flow as a function of voltage (u- $V$ characteristic) with various distance between the electrodes $(d=4,6,8,10,12$ and $14 \mathrm{~mm})$ of the electrode pin-multi concentric rings configuration (UT) and pin-single ring configuration (US) is shown in Figure 2 (a) and (b)

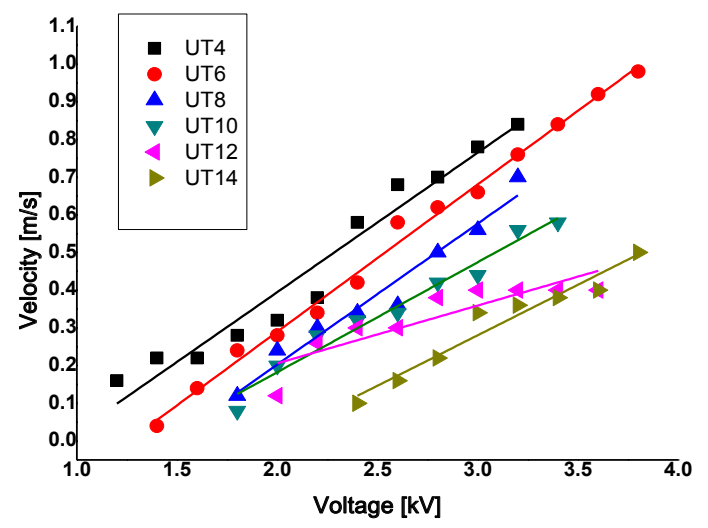

(a)

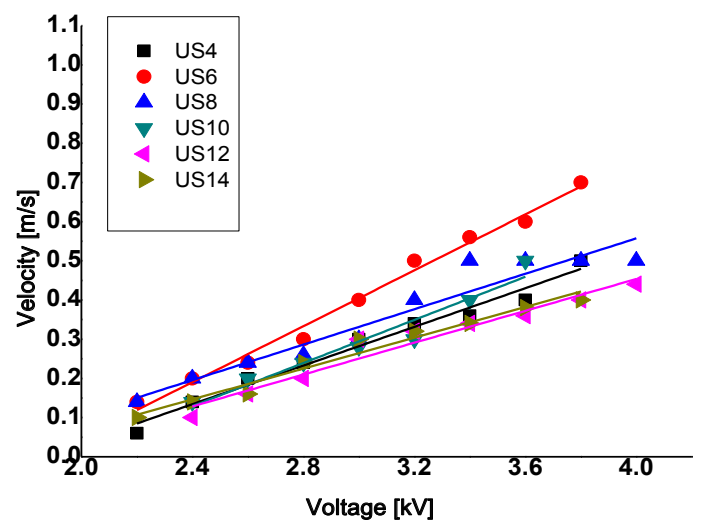

(b)

Fig.2. $\mathbf{u}-V$ Characteristics of (a) pin-multi concentric rings and (b) pin-single ring electrode configuration

In Figure 2.(a) and (b) it appears that in all of the distance between the electrodes $(d)$, the greater the applied voltage the greater the velocity of the EHD flow. This is because the relationship between the voltage and the velocity of the EHD flow is a linear function. In accordance with the theoretical formulation by Goodenough et al [15] which shows that $\mathbf{u} \infty \quad V$. However, variations in the distance between the electrodes $(d)$ at a fixed voltage shows that the greater the distance between the electrodes, the smaller the velocity of the EHD flow. This happens because the electric field strength is a function of the distance between the electrodes. The greater the distance between the electrodes, the electric field strength is getting smaller.

\subsection{The Velocity Comparison of the EHD flow}

It is very important to know the comparison between the EHD flow velocity for electrode configuration of pin-multi concentric rings (uT) with electrode configuration of pin-single ring (uS). The EHD flow velocity in pin-multi concentric rings electrodes (UT) and in pin-single ring electrode (uS) ratio as a function of the distance between the electrodes $(d)$ at various the voltages $(V)$ is shown in Figure 3. It is shown that the value of $((\mathbf{u T}) /(\mathbf{u S}))$ at all $d$ value is greater than 1 (one).

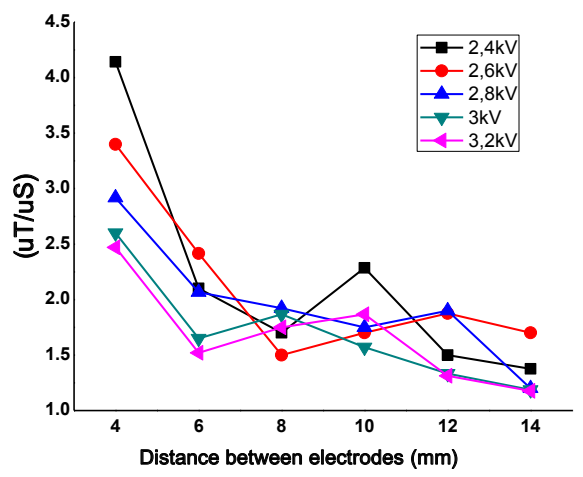

Fig.3. Graph (uT/uS) function of the distance between the electrodes $(d)$ on voltage $(V)$ remain

It can be concluded that at the same electrodes distance $d$ the velocity of EHD flow for uT is greater than the velocity of EHD flow for $\mathbf{u S}$ at any voltage. This is due to the electric flux density in the electrode pair of pin-multi concentric rings is greater than that in pin-single ring electrode pair. The large electric flux density will increase the ion density. The ions move towards multi-concentric ring electrodes and collide with neutral air molecules with very high collision frequency. The momentum transfer of positive ions to molecules of gas in the area between the electrodes is very large and in turn this will increase the EHD flow velocity.

\section{3 u-P Characteristic of EHD Flow}

Figure 4 shows the graph of $\mathbf{u}-P$ characteristics of EHD flow in pin-multi concentric rings electrodes and pinsingle ring electrode. The greater the electric power results in the greater velocity of the EHD flow (u) produced. This is because the velocity is proporsional to $P^{1 / 3}$. This is in accordance with the results of studies conducted by Goodenough et al [15] and Komeilia et al [16]. The initial electrical power that produced the initial 
velocity of EHD flow in pin-multi concentric rings electrodes configuration is smaller than that in the pinsingle ring electrode. At the same electrical power, eg at $P=100 \mathrm{~mW}$, the velocity of EHD flow in pin-multi concentric rings electrode configuration will be larger than that in the pin-single ring configuration. This is in accordance with the $\mathbf{u}-V$ characteristics.

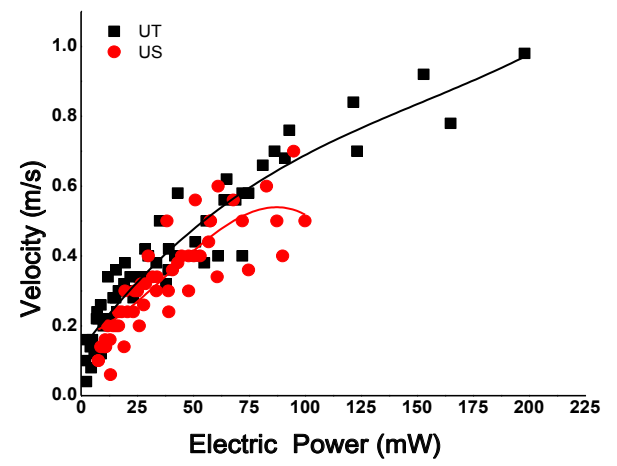

Fig.4. $\mathbf{u}-P$ Characteristics of EHD flow in pin-multi concentric rings electrodes and pin-single ring electrodes.

Figure 4. represents more obvious that $\mathbf{u} \sim P^{1 / 3}$. The velocity of EHD flow in configuration of pin-multi concentric rings $\mathrm{uT}$ is always greater than the velocity of EHD flow in pin-single ring configuration $\mathrm{uS}$. The value of uT can reach $0.9 \mathrm{~m} / \mathrm{s}$ for a power of $200 \mathrm{~mW}$, before arc discharge which is characterized by extremely high currents and the voltage is reduced to be near zero. In the uS, the electric power of $100 \mathrm{~mW}$ is still possible to be applied before reaching the arc discharge to produced velocity of EHD flow of $0.7 \mathrm{~m} / \mathrm{s}$.

From the aspect of electric power, the EHD flow generator with pin-multi concentric rings configuration can be operated with a higher power than pin-single ring configuration. This is due to the stability (not easily occur arc) of the pin-multi concentric rings.

\section{Conclusion}

The velocity of EHD flow as a function of the voltage at a certain distance between electrodes showed that the EHD flow velocity is proportional to the voltage and proportional to $P^{1 / 3}$. At the same distance between the electrodes, the velocity of EHD flow for pin-multi concentric rings is always greater than for pin-single ring at any voltages.

\section{References}

1. M. Nur., A. H. Azzulkha, M. Restiwijaya, Z. Muchlisin and Sumariyah, The Study of Electrohydrodynamic and Wind Ions Direction, Produced by Positive Corona Plasma Discharge, Advances in Physics Theories and Applications ISSN 2224-719X (Paper) ISSN Vol. 33, 2225-0638 (Onlines) (2014)
2. N. St. J. Braithwaite, Introduction to gas discharges, Plasma Sources Sci. Technol. 9, $517-$ 527 (2000)

3. E..D. Fylladitakis, M.P. Theodoridis and A.X. Moronis, Review on the History, Research, and Applications of of Electrohydrodynamics, IEEE, 0093-3813 (2014)

4. A. Repanas, S. Andriopoulou, B. Glasmacher, The significance of electrospinning as a method to create fibrous scaffolds for biomedical engineering and drug delivery applications, Journal of Drug Delivery Science and Technology 31, 137-146 (2016)

5. P. Sofokleous, E. Stride, W. Bonfield, M. Edirisinghe, Design, construction and performance of a portable handheld electrohydrodynamic multineedle spray gun for biomedical applications, Materials Science and Engineering 33, 213-223 (2013)

6. I.Y. Chen, M.Z. Guo, K.S. Yang, C. C. Wang, Enhanced cooling for LED lighting using ionic wind, International Journal of Heat and Mass Transfer 57, 285-291 (2013)

7. A.H. Hosseinloo, S. P. Tan, F. F. Yap, K. C. Toh , Shock and vibration protection of submerged jet impingement cooling systems: Theory and experiment Applied Thermal Engineering 73, 10761086 (2014)

8. E. Bardy, M. Hamdi, M. Have, O. Rouaud, Transient exergetic efficiency and moisture loss analysis of forced convection drying with and without electrohydrodynamic enhancement, Energy 89, 519-527 (2015)

9. A. Martynenko, W. Zheng, Electrohydrodynamic drying of apple slices: Energy and quality aspects, Journal of Food Engineering 168, 215-222 (2016)

10. M. C. Karam, J. Petit, D. Zimmer, E.B. Djantou, J. Scher, Effects of drying and grinding in production of fruit and vegetable powders: A review, Journal of Food Engineering 188, 32-49 (2016)

11. J.I. Zhang and and F.C. Lai, Effect of emitting electrode number on the performance of EHD gas pump in a rectangular channel, Journal of Electrostatics 69, 486-493 (2011)

12. Sumariyah, Kusminarto, Arief H., Pekik N., Zaenul M., and Evi S., Modeling of Electric Potential Distribution in EHD Flow Zone Utilizing Pin-Multi Ring Consentric Electrodes, Procedia Environmental Sciences 23, 260 - 265 (2015)

13. Sumariyah, Kusminarto, Arief H., and Pekik N., Velocity Measurement of EHD Flow Produced by Pin-Multi Concentric Ring electrodes Generator, Applied Mechanics and Materials Vol 271, pp 227231 (2015)

14. Sumariyah, Kusminarto, Arief H. and Pekik N., Determination of ion mobility in EHD flow zone of plasma generator, International Conference of Chemical and Material Engineering (ICCME) AIP Conf. Proc. 1699, 040019-1-040019-5 (2015)

15. Timothy I.J. Goodenough, Peter W. Goodenough, Sylvia M. Goodenough, The efficiency of corona 
wind drying and its application to thefood industry, Journal of Food Engineering 80, 1233-1238 (2007)

16. B. Komeilia, J.S. Changa,G.D.Harvela C.Y. Chingc, D. Brociloa, Flow characteristics of wirerod type electrohydrodynamic gas pump under negative corona operations, Journal of Electrostatics 66, 342-353 (2008) 